\title{
25 Research Soure \\ Prediction of Potential Prognostic Tumor Markers of Re-ascites After Chemotherapy for Ovarian Cancer Based on LC/MS Method
}

Miao Liu

Harbin Medical University

Yu Liu

Harbin Medical University

Yixin Jing

Harbin Medical University

Shuang Zhao

Tumor Hospital of Harbin Medical University

Shujia Yang

Harbin Medical University

Nan Zhang

Harbin Medical University

Shi Jin

Harbin Medical University

Yafei Li

Tumor Hospital of Harbin Medical University

Mingjiao Weng

Harbin Medical University

Yongheng Yang

Harbin Medical University

Hua Feng

Harbin Medical University

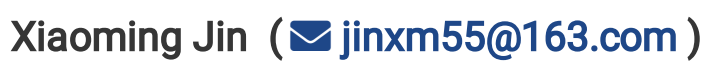

Harbin Medical University https://orcid.org/0000-0003-1531-3803

Dan Kong

Tumor Hospital of Harbin Medical University

\section{Research}

Keywords: Ovarian cancer, ovarian cancer ascites, metabonomics, differential metabolites, tumor markers

Posted Date: August 27th, 2020 
DOI: https://doi.org/10.21203/rs.3.rs-64922/v1

License: (c) (i) This work is licensed under a Creative Commons Attribution 4.0 International License. Read Full License 


\section{Abstract}

Background: Tumor cells are not only in a state of exuberant metabolism, but also inseparable from the surrounding microenvironment for the occurrence and development of tumors. By means of metabonomics technology, the metabolic profiles of re-ascites after chemotherapy in ovarian cancer ascites microenvironment were compared, and new tumor markers were screened for the treatment and prognosis of ovarian cancer.

Methods: Thirty-seven cases of ovarian cancer were selected, of which 12 cases had ovarian cancer tissues, ascites supernatant and precipitated cells. By using UHPLC-Q-TOF MS/MS and combined with HMDB database, differential metabolites are identified and then differential pathways are enriched.

Results: The results revealed that 10 differential metabolites were screened between chemotherapy and nochemotherapy groups, and 9 differential metabolites were found in chemotherapy-sensitive and chemotherapy-resistant groups, in the ascites of ovarian cancer. Among the 19 differential metabolites, 9 metabolites combined with serum CA125 were analyzed by receiver operating characteristic analyses, which showed more the predictive value of diagnosis of malignancy. Among them, Tow differential metabolites (2/9) were correlated with the expression of CA125, five differential metabolite (5/9) related enzyme genes were statistically significant in overall survival and progression free survival (PFS) analysis. Four differential metabolites (4/9) were correlated with the expression of uric acid, creatinine, $\gamma$-GGT and ALP in serum.

Conclusions: The results suggest that differential metabolites are mainly involved in PPAR signal pathway, steroid biosynthesis, fatty acid metabolism, secondary bile acid biosynthesis and so on. Lipid metabolites of ovarian cancer ascites cells may promote fatty acid oxidation by activating AMPK, promote lipid synthesis through mTOR/PPAR, provide energy for cancer cells, inhibit apoptosis and promote metastasis. Thus targeted lipid metabolism signal transduction axis can inhibit ovarian cancer metastasis and drug resistance.

\section{Introduction}

Ascites formation is a common complication of advanced ovarian cancer [1]. Some patients still produce ascites repeatedly after chemotherapy, indicating that they are resistant to chemotherapy. At present, there is a lack of clinical markers related to chemosensitivity or drug resistance, obtained from ascites. Therefore, it is very important to find potential tumor markers that can predict the efficacy and drug resistance of platinum chemotherapy for ovarian cancer.

Cancer is a metabolic disease characterized by metabolic reprogramming of cells, which is essential to maintain a high proliferation rate and resist changes in cell death signals [2,3]. Metabonomics is a rapid and effective method to identify new cancer biomarkers, which has gradually become a supplementary technology of genomics and proteomics. It integrates high-throughput, high-resolution analysis technology with bioinformatics to conduct research on biology, and at the metabolic level. It provides a unique perspective for understanding biology, and its applications in tumor research are becoming increasingly mature and has become a hot spot in domestic and foreign research [4, 5]. It promotes the discovery of 
biomarkers in disease and drug treatment targets. In addition, it can also provide valuable information about drug resistance and disease recurrence [6], The application of this technology can help discover new targets

for the treatment of ascites for ovarian cancer.

In this study, ascites and cancer tissues were divided into no-chemotherapy and chemotherapy, chemotherapy-sensitive and chemotherapy-resistant groups. we performed Ultra-performance liquid chromatography quadrupole time-of-flight mass spectrometry (UHPLC/Q-TOF MS/MS) metabonomics to analyze the characteristics of metabolites between the two groups and the relationship between differential metabolites and platinum efficacy and to look forward to providing new clues and new hopes in guiding clinical diagnosis and treatment.

\section{Materials And Methods}

\section{Collection of ascites and tissue samples from ovarian cancer}

82 cases of high-grade serous ovarian cancer ascites from November 2014 to June 2019 from the Harbin Medical University Affiliated Cancer Hospital were collected. 37 ascites samples were selected and divided into non-chemotherapy group (No chemotherapy, N) and platinum chemotherapy group (Chemotherapy, C). The patients who underwent chemotherapy were further divided into the chemo-sensitive group ( $\geq 6$ months for platinum sensitivity, CS) and the chemo-resistant group ( $<6$ months is platinum resistance, CR) [7]. We also acquired 22 fresh ovarian cancerous tissue samples of the 37 cases. Among them, 12 cancer tissues can correspond to the ascites supernatants and precipitated ascites cells to form self-pairing (Table S1). All cases had complete clinicopathological data and written informed consent.

\section{processing of ascites and cancer tissue}

The supernatants of cancerous ascites were collected into $1.5 \mathrm{~mL}$ centrifuge tubes. Then, moderate ACK lysis (Leagene Biotechnology, China) buffer was added to the precipitates, which were collected into $1.5 \mathrm{~mL}$ centrifuge tubes. The paraffin blocks of ovarian cancer tissues were cut into $20 \mu \mathrm{m}$ and stored at $-80^{\circ} \mathrm{C}$. Experimental details about metabolite extraction and liquid chromatography-mass spectrometry (LC-MS) data acquisition are provided in the Supporting information.

\section{Data Processing}

Raw MS data (.d) files are preprocessed by XCMS online [8] and MetaboAnalyst 4.0 [9]. The generated data matrix consisted of the mass-to-charge ratio $(\mathrm{m} / \mathrm{z})$ value, retention time (RT), and peak abundance.

Multivariate statistical analysis (PCA and PLS-DA) was carried out by SIMCA-P software package (14.0). The validity of the model is tested by replacement to avoid the overfitting. The variable important in the projection (VIP) value was calculated for each variable in the PLS-DA model. In addition, An independent t-test was used for comparison. The differential metabolites with $p$ value less than 0.05 and VIP value greater than 1 were selected. The combination of accurate mass and experimental MS/MS spectra are matched with the 
structural information of metabolites in human metabolite database (HMDB). Dysregulated metabolites were mapped into the KEGG database for pathway enrichment analysis using the hypergeometric test.

\section{Statistical Analyses}

Clinical data are represented as mean \pm sd. Statistical analyses were performed using SPSS 21.0 software. Spearman's test was used to evaluate the associations between differential metabolite and serum biochemical index. Comparisons between two or multiple parameters were performed using the chi-square test. A p value less than 0.05 was set as the significance level. Kaplan-Meier analysis is used to evaluate the prognosis of patients with ovarian cancer. Random forest analysis estimates the importance of each metabolite in MetaboAnalyst 3.0. The receiver operating characteristic (ROC) was used to evaluate the diagnostic performance of metabolite biomarkers.

\section{Results}

\section{Metabonomics analysis of ovarian cancer tissue and ascites}

The metabonomic data were visualized by unsupervised principal component analysis(PCA). In 37 cases of ascites supernatant, 23 cases of precipitated cells and 22 cases of cancer tissue samples, QC samples in PCA plots were clustered tightly (Fig. S1), which indicated that the data quality was very good. In a supervised model of PLS-DA, ascites and tissue samples were clearly separated between $\mathrm{N}$ and $\mathrm{C}, \mathrm{CS}$ and $\mathrm{CR}$, indicating that all the three groups had different metabolic profiles and there was no over-fitting (Fig. 1).

\section{Select differential metabolites in ovarian cancer tissues and ascites}

\section{Metabolic characteristics of supernatant, precipitated cells and cancer tissues in ovarian cancer}

A total of 7579, 10053 and 11053 metabolic peaks were detected in 37 cases of ascites supernatant, 23 cases of precipitated cells and 22 cases of ovarian cancer, respectively (Fig. 2a). Heatmap and correlation analysis showed that metabolites were well distinguished in no-chemotherapy, chemosensitivity and Chemotherapy resistance groups, and there was a certain correlation between them (Fig. 2b). It is suggested that there may be different metabolic patterns in N vs C and CS vs CR and the metabolites promote or inhibit each other.

\section{Analysis of differential metabolites and common differential metabolites in each sample set}

Differential metabolites were screened in N vs C and CS vs CR for each sample set(Table S2-3). The corresponding coexisting metabolites of differential metabolites in other samples of each group were 49, 31 and 9 , respectively (Table 1 ). All the differential metabolites were combined and the differential metabolites with higher significance were screened by random forest analysis ( $n=15$, respectively (Fig. $2 c)$. The coexistence metabolites were intersected with random forest results in N vs C and CS vs CR, respectively. Screening shared metabolites in co-existing metabolites and random forests, and further combining them (Fig. 2d). 10 and 9 differential metabolites were obtained in N vs C and CS vs CR, respectively. 
Table 1

Results of different metabolites in ovarian cancer tissues, ascites supernatant and precipitated cells

\begin{tabular}{|c|c|c|c|c|c|c|c|c|c|c|}
\hline & \multicolumn{4}{|c|}{37 unpaired $\operatorname{cases}(n=82)-I$} & \multicolumn{4}{|c|}{12 paired cases $(n=44)-I I$} & \multicolumn{2}{|c|}{$\begin{array}{l}12 \text { paired cases }(n= \\
36)-I I I\end{array}$} \\
\hline & \multicolumn{2}{|c|}{ different } & \multicolumn{2}{|c|}{ coexisting } & \multicolumn{2}{|c|}{ different } & \multicolumn{2}{|c|}{ coexisting } & \multirow{2}{*}{$\begin{array}{l}\text { different } \\
\text { N vs C }\end{array}$} & \multirow{2}{*}{$\begin{array}{l}\text { coexisting } \\
\mathrm{N} \text { vs C }\end{array}$} \\
\hline & $\begin{array}{l}\mathrm{N} \\
\text { vs } \\
\mathrm{C}\end{array}$ & $\begin{array}{l}\text { CS vs } \\
\text { CR }\end{array}$ & $\begin{array}{l}\mathrm{N} \\
\text { vs } \\
\mathrm{C}\end{array}$ & $\begin{array}{l}\text { CS vs } \\
\text { CR }\end{array}$ & $\begin{array}{l}\mathrm{N} \\
\text { vs } \\
\mathrm{C}\end{array}$ & $\begin{array}{l}\text { CS vs } \\
\text { CR }\end{array}$ & $\begin{array}{l}\mathrm{N} \\
\text { vs } \\
\mathrm{C}\end{array}$ & $\begin{array}{l}\text { CS vs } \\
\text { CR }\end{array}$ & & \\
\hline supernatant & 26 & 20 & 13 & 14 & 18 & 4 & 10 & 2 & 7 & 5 \\
\hline cells & 6 & 10 & 2 & 2 & & & & & 3 & 1 \\
\hline tissues & 14 & 16 & 10 & 8 & 14 & 16 & 10 & 9 & 7 & 3 \\
\hline Total & 46 & 46 & 25 & 24 & 32 & 20 & 20 & 11 & 17 & 9 \\
\hline sum & 92 & & 49 & & 52 & & 31 & & 17 & 9 \\
\hline
\end{tabular}

Note: 37 cases of unmatched-I group, 22 cases of paired-II group, 12 cases of paired-III group; bold text means co-existing metabolites.

\section{Correlation between differential metabolites and clinical pathological data}

\section{ROC analysis of differential metabolites combined with serum CA125}

ROC analysis showed that the distinguishing ability of metabolites was less than that of CA125, but when combined with CA125, nine metabolites increased. They are carnosine, 20-COOH-leukotriene E4 (20-COOHLTE4), sebacic acid in N vs C; 1,25-dihydroxyvitamin D3-26,23-lactone (1,25-Lactone), 20a,22bdihydroxycholesterol, 3a,6a,7b-trihydroxy-5b-cholicacid (Muricholic acid), 3a,7a,12a,19-tetrahydroxy-5b-cholic aci $(3,7,12,19-$ THCA), dihydrothymine, hexadecanoic acid in CS vs CR (Fig. 3a). The combination of metabolites and CA125 may improve the predictive value of ovarian cancer diagnosis.

\section{Correlation between differential metabolites and clinical serum biochemical indexes in patients}

The serum biochemical indexes of 82 patients with ovarian cancer showed that serum CA125, uric acid and a-hydroxybutyrate dehydrogenase (HBDH) were significantly decreased, $y$-glutamyl transpeptidase ( $\mathrm{Y}$-GGT) was significantly increased in chemotherapy group, serum CA125, creatinine, lactate dehydrogenase (LDH), alkaline phosphatase (ALP) were increased, and total cholesterol was significantly decreased in Chemotherapy resistance group ( $p<0.05$, Fig. 3b). Correlation analysis showed that 20-COOH-LTE4 and hexadecanoic acid were positively correlated with CA125 $(p<0.05), 20-\mathrm{COOH}-\mathrm{LTE} 4$ was negatively correlated with creatinine $(p<0.05)$, carnosine was positively correlated with uric acid $(p<0.05), 20 \mathrm{a}-22 \mathrm{~b}$ dihydroxycholesterol was positively correlated with $Y-G G T$ and ALP, hexadecanoic acid was positively correlated with $\mathrm{Y-GGT}$ and $\mathrm{HBDH}$, and other metabolites were not significantly correlated with serum biochemical indexes (Fig. 3c). 


\section{Survival analysis of genes related to differential metabolites}

Progression free survival analysis (PFS) showed that the high expression of enzyme gene had a poor prognosis, which were CNDP1 (carnosine), LTC4S (20-COOH-LTE4), CYP11A1 (20a,22b-dihydroxy cholesterol), CYP7A1 (W-Muricholic acid), CYP27A1 (3,7,12,19-THCA) ( $p<0.05$, Fig. 4a). Low expression has a poor prognosis, which are ACADL (sebacic acid), DHCR24 (1,25-lactone), AGXT2 (dihydrothymine), ACSL1 (hexadecanoic acid) ( $p<0.05$, Fig. 4b). Elevated carnosine in no-chemotherapy group is also higher in patients with poor progression free survival. Carnosine that is increased in no chemotherapy group is also higher in patients with poor disease-free survival. Similarly, among drug-resistant patients, increased 20a, 22b-dihydroxycholesterol is also higher in patients with poor progression-free survival, and decreased 1,25lactone, dihydrothymine and hexadecanoic acid are also lower in patients with poor progression-free survival. In addition, LTC4S, CYP11A1, CYP27A1, ACSL1, and AGXT2 all showed significance in overall survival and progression-free survival analysis. It suggests that 20a, 22b-dihydroxycholesterol, 1,25-lactone, dihydrothymine, and hexadecanoic acid may become the most promising signs for judging whether ovarian cancer develops drug resistance Things.

\section{Pathway analysis of differential metabolites in ovarian cancer tissue and ascites}

Metabolite pathway analysis showed that 25 and 12 pathways were enriched in N vs C, CS vs CR (Table S45), and there were 6 common pathways (Fig. 5a-b). Differential metabolites and clinical serological indicators also participate in the same pathway, including arachidonic acid, purine, arginine, folate metabolism and steroid synthesis, etc. The change trend of metabolic pathways is consistent. This may explain that changes in metabolites in cancer tissue not only lead to changes in ascites metabolites, but also cause serological changes.

\section{The potential mechanism of differential metabolites in the progression of ovarian cancer}

For 51 genes of 9 metabolite-related enzymes, STRING protein interaction network analysis (Fig. 5c) was performed, which has significant correlation $(p<0.001)$. KEGG enrichment analysis shows that it is mainly involved in the PPAR signaling pathway, steroid biosynthesis, fatty acid metabolism, secondary bile acid biosynthesis, et al. Molecular pathways that may be involved in ovarian cancer are constructed (Fig. 5d). Metabolites and related enzymes may play a role in the progression of ovarian cancer and the development of drug resistance, including malignant proliferation, invasion, apoptosis, et al. (Table 2). 
Table 2

Biological function of potential biomarkers and related enzyme genes in ovarian cancer

\begin{tabular}{|c|c|c|c|c|c|c|c|}
\hline Metabolites & $\begin{array}{l}\text { Log } \\
\text { FC }\end{array}$ & $\begin{array}{l}\text { CA125 } \\
+ \text { AUC }\end{array}$ & Gene & $\begin{array}{l}\text { KM p- } \\
\text { value }\end{array}$ & $\begin{array}{l}\text { poor } \\
\text { survial }\end{array}$ & pathway & faction \\
\hline Carnosine & 0.65 & 0.884 & CNDP1 & $\begin{array}{l}< \\
0.001\end{array}$ & high & $\begin{array}{l}\text { Histidine } \\
\text { metabolism }\end{array}$ & Apoptosis \\
\hline 20-COOH-LTE4 & -0.28 & 0.899 & LTC4S & 0.012 & high & $\begin{array}{l}\text { Arachidonic } \\
\text { acid } \\
\text { metabolism }\end{array}$ & Angiogenesis \\
\hline Sebacic acid & 0.2 & 0.879 & ACADL & $\begin{array}{l}< \\
0.001\end{array}$ & low & $\begin{array}{l}\text { Fatty acid } \\
\text { metabolism }\end{array}$ & $\begin{array}{l}\text { Inhibit } \\
\text { metastasis }\end{array}$ \\
\hline Calcitriol lactone & -0.66 & 0.963 & DHCR24 & 0.011 & low & $\begin{array}{l}\text { Steroid } \\
\text { biosynthesis }\end{array}$ & $\begin{array}{l}\text { Inhibit } \\
\text { proliferation }\end{array}$ \\
\hline $\begin{array}{l}\text { 20a,22b- } \\
\text { Dihydroxycholesterol }\end{array}$ & 0.3 & 0.88 & CYP11A1 & 0.046 & high & $\begin{array}{l}\text { Steroid } \\
\text { hormone } \\
\text { biosynthesis }\end{array}$ & Proliferation \\
\hline Muricholic acid & -0.28 & 0.944 & CYP7A1 & 0.032 & high & $\begin{array}{l}\text { bile acid } \\
\text { biosynthesis }\end{array}$ & $\begin{array}{l}\text { Proliferation } \\
\text { drug } \\
\text { resistance }\end{array}$ \\
\hline 3,7,12,19-THCA & -0.47 & 0.935 & CYP27A1 & 0.01 & high & $\begin{array}{l}\text { bile acid } \\
\text { biosynthesis }\end{array}$ & $\begin{array}{l}\text { Proliferation } \\
\text { drug } \\
\text { resistance }\end{array}$ \\
\hline Dihydrothymine & -0.48 & 0.88 & AGXT2 & 0.034 & low & $\begin{array}{l}\text { Pyrimidine } \\
\text { metabolism }\end{array}$ & $\begin{array}{l}\text { Anti- } \\
\text { apoptosis } \\
\text { drug } \\
\text { resistance }\end{array}$ \\
\hline Hexacosanoic acid & -0.14 & 0.926 & ACSL1 & 0.018 & low & $\begin{array}{l}\text { Fatty acid } \\
\text { metabolism }\end{array}$ & $\begin{array}{l}\text { Proliferation } \\
\text { drug }\end{array}$ \\
\hline
\end{tabular}

\section{Discussion}

At present, metabonomics is applicated in a variety of tumors [10-13]. Biological samples, including urine, plasma and tumor tissue, have been applied to metabolism-based ovarian cancer research [14-17]. It is often used to distinguish between healthy and ovarian cancer patients, ovarian malignant tumors and borderline tumors, and can also be used to detect recurrent tumors. Compared with urine and plasma, ascites is highly selective in filtering and concentrating plasma metabolites through the serosa. Therefore, ascites may become an ideal fluid for the study of metabonomics for the discovery of biomarkers $[18,19]$. Recurrent ascites and rapid resistance to platinum therapy are common in advanced ovarian cancer. The five-year survival rate of EOC has been around 30\% [20]. Metabolomic profiling of human malignant effusion remain a field poorly investigated. At present, there are few studies on predicting chemotherapy resistance. In this study, metabonomic analysis was carried out on platinum sensitive and drug resistant cancer tissues and ascites in order to discover the new biomarkers to monitor disease progression and drug resistance for precise treatment. 
It was observed that there were different expression profiles in patients no-chemotherapy, chemo-sensitive and chemo-resistant. Cancer cells exhibited increased lipid dependency during growth and proliferation. The high free fatty acid content in ascitic fluid provides a huge energy source and may force ovarian cancer cells to undergo metabolic reprogramming from aerobic glycolysis to fatty acid $\beta$-oxidation to produce energy for tumor growth [21]. Compared with chemotherapy samples (ascites and tissues), the levels of saturated/unsaturated fatty acids (sebacic acid囚20-COOH-LTE4), glycerol phospholipids(PC\PGP), steroids (7-Dehydrocholesterol $\square$ Squalene[Ergosterol) and amino acid (2-Aminomuconic acid[L-Tryptophan $\square$ Formiminoglutamic acid) metabolites were down-regulated in untreated samples. Consistent with Anna, et al. [18]. It was confirmed that the decrease of lipids and essential amino acids was directly related to tumor metabolism and promoted cell proliferation and growth. According to the analysis of coexisting metabolites in ascites and cancer tissues, nine metabolites were selected as possible biomarkers of ovarian cancer. The AUC values of nine metabolites combined with CA125 were greater than 0.875 , which showed high diagnostic ability.

20-COOH-LTE4, a class of inflammatory liposomes synthesized by arachidonic acid, is often used to evaluate mast cell activation22. Recently, it has been found that $[22,23]$ leukotriene E4 (LTE4) can also induce prostaglandin D2 (PGD2) production in human mast cells by activating peroxisome proliferator receptor (PRPP) and inhibit the growth of cancer cells. It is an independent indicator of good prognosis. Sebacic acid is a saturated, straight-chain naturally occurring dicarboxylic acid. deficiency of medium chain acyl-CoA dehydrogenase (ACADM) results in the inhibition of $\beta$-oxidation and indirectly leads to the increase of sebacic acid. This study found that sebacic acid increased in chemotherapy ascites, which may be related to the inhibition of ACADM activity. Abnormal expression of ACADM was also found in hepatocellular carcinoma [24]. It promotes cell proliferation, migration and invasion by regulating fatty acid metabolism. Therefore, it is suggested that ACADM is expected to be a therapeutic target.

Compared with the sensitive group, the recurrent group also showed significant changes in fatty acid metabolism, in which hexadecanoic acid increased significantly in drug resistant ascites. Hexadecanoic acid is a very long-chain fatty acid, which was once considered as a sign of peroxisome metabolism disorder. However, Adriana et al. [25] proposed that cetanoic acid is the product of fatty acid prolongation in tumor tissue. Fatty acid prolonging enzyme overexpression [26] promotes peroxisome $\beta$ oxidation, relatively increases fatty acid uptake and utilization, and promotes drug resistance. It is consistent with the plasma study of recurrent ovarian cancer by Ke. et al. [27].

In addition, the four metabolites in the drug resistant group were all involved in the metabolism of 7 dehydrocholesterol. String protein interaction analysis also showed a significant correlation. Emerging evidence has suggested [28] that a high-fat diet and maladjusted WNT signals synergistically increased intestinal secondary bile acid levels in colorectal cancer. It also can activate proteasome to degrade p53 and promote the production of drug resistance. This study found that 20a-22b-dihydroxycholesterol was increased in the resistant group, and CYP11A1 can convert it into pregnenolone indirectly. The latest research shows that CYP11A1 exerts its anti-tumor activity through vitamin D substitution [29]. At the same time, this study also found that 1,25-lactone was down-regulated in the drug-resistant group. This consistency can confirm the beneficial role of vitamin D in cancer prevention and treatment. These results suggest that 
cholesterol, as a precursor of bile acid and steroid synthesis, promotes cancer cell proliferation, invasion and inhibition of apoptosis [30]. In a word, dynamic lipid metabolism changes occur in advanced ovarian cancer cells to adapt to the changes of nutritional microenvironment.

Studies have shown that [31] diabetes is a risk factor for many cancers, especially ovarian cancer. In this study, the incidence of diabetes was higher in the drug resistant group. Long-term hyperglycemia can promote the growth of tumor cells. In turn, it can promote the expression of glycolysis-related enzymes such as lactate dehydrogenase (LDH) [32]. In this study, it was also found that LDH, ALP and y-GGT were upregulated in drug-resistant group, and $\mathrm{HBDH}$ was up-regulated in non-chemotherapy group. These metabolic enzymes that promote malignant transformation are expected to become therapeutic targets.

Ovarian cancer cells utilize lipid metabolism in the ascites or omental microenvironment through AMPK/ACC/FASN-mediated lipogenesis and mTOR /TAK1 /NF-KB signaling cascades, which in turn, enhanced ovarian cancer metastasis and aggressiveness [21]. Cholesterol can promote angiogenesis of tumor cells through mTOR signal [33]. The signal transduction network in which metabolites may be involved needs further study.

\section{Conclusions}

In this study, the metabolic changes of paired ascites and tissues during chemotherapy and relapse resistance were analyzed for the first time, and the potential differential metabolites in ascites were screened to reflect the efficacy of chemotherapy and monitor drug-resistant recurrence. The biomarkers of diagnosis and prognosis of ovarian cancer were screened by metabonomics technology to provide new ideas and new hope for judging the prognosis of ovarian cancer ascites and revising the treatment plan in time.

\section{Abbreviations}

ALP alkaline phosphatase

AUC Area under the ROC curve

CHOL Cholesterol

GLU Glucose

CREA creatinine

HBDH a-hydroxybutyrate dehydrogenase

HMDB Human metabolome database

KEGG Kyoto encyclopedia of genes and genomes

LDH lactate dehydrogenase 
MS/MS Tandem mass spectroscopy

PFS Progression free survival

QC Quality control

QTOF Quadrupole time-of-flight

RF Random forest

ROC Receiver operating characteristic curve

TRIG Triglyceride

UA uric acid

UPLC Ultra-performance liquid chromatography

VIP Variable importance in the projection

Y-GGT Y-glutamyl transpeptidase

\section{Declarations}

\section{Ethics approval and consent to participate}

This study was approved by the ethics committee of The Third Affiliated Hospital of Harbin Medical University, and wrote informed consent was obtained from all patients. This study was approved by the ethics committee of Harbin Medical University.

\section{Consent for publication}

Not applicable.

\section{Availability of data and materials}

The datasets supporting the conclusions of this article are included within the article and its additional files.

\section{Competing interests}

The authors declare that they have no competing interests.

\section{Funding}

This work was supported by Distinguished Young Fund of Tumor Hospital of Harbin Medical University (JCQN2019-03); Haiyan Foundation of Tumor Hospital of Harbin Medical University (JJZD2019-07). Joint guidance Project of Natural Science Foundation of Heilongjiang Province (LH2020H123). 


\section{Authors' Contributions}

X.-M. Jin and D. Kong designed the research study and interpreted the data. D. Kong 『S. Jin, Y.-F. Li, M.-J. Weng and $\mathrm{H}$. Feng collected samples and clinical-pathological data. M. Liu, Y. Liu, Y.-X. Jing, Y.-H. Yang, S.-J. Yang and N. Zhang performed the experiments. M. Liu, Y.-X. Jing, Y.-H. Yang and S. Jin analyzed Metabonomic data. X.-M. Jin, D. Kong, M. Liu and Y. Liu wrote the manuscript. All authors have read and approved the final manuscript.

\section{Acknowledgements}

The authors thank the metabolic platform Key Laboratory of Myocardial Ischemia, second affiliated Hospital of Harbin Medical University for expert technical assistance. We would like to thank Department of Pathology of Harbin Medical University, Department of Gynecology of Tumor Hospital of Harbin Medical University.

\section{References}

1. Han Q, Huang B, Huang Z, Cai J, Gong L, Zhang Y, et al. Tumor cellfibroblast heterotypic aggregates in malignant ascites of patients with ovarian cancer. Int J Mol Med. 2019;44(6): 2245-2255.

2. Wishart DS. Metabolomics for Investigating Physiological and Pathophysiological Processes. Physiol Rev. 2019;99(4): 1819-1875.

3. Wang YP, Li JT, Qu J, Yin M, Lei QY. Metabolite sensing and signaling in Cancer. J Biol Chem. 2020.

4. Antony F, Deantonio C, Cotella D, Soluri MF, Tarasiuk O, Raspagliesi F, et al. High-throughput assessment of the antibody profile in ovarian cancer ascitic fluids. Oncoimmunology. 2019;8(9): e1614856.

5. Chen J, Hou H, Chen H, Luo Y, Zhang L, Zhang Y, et al. Urinary metabolomics for discovering metabolic biomarkers of laryngeal cancer using UPLC-QTOF/MS. J Pharm Biomed Anal. 2019;167: 83-89.

6. Cao Y. Adipocyte and lipid metabolism in cancer drug resistance. J Clin Invest. 2019;129(8): 3006-3017.

7. Cortez AJ, Tudrej P, Kujawa KA, Lisowska KM. Advances in ovarian cancer therapy. Cancer Chemother Pharmacol. 2018;81(1): 17-38.

8. Forsberg EM, Huan T, Rinehart D, Benton HP, Warth B, Hilmers B, et al. Data processing, multi-omic pathway mapping, and metabolite activity analysis using XCMS Online. Nat Protoc. 2018;13(4): 633651.

9. Chong J, Soufan O, Li C, Caraus I, Li S, Bourque G, et al. MetaboAnalyst 4.0: towards more transparent and integrative metabolomics analysis. Nucleic Acids Res. 2018;46(W1): W486-W494.

10. Wu JY, Huang TW, Hsieh YT, Wang YF, Yen CC, Lee GL, et al. Cancer-Derived Succinate Promotes Macrophage Polarization and Cancer Metastasis via Succinate Receptor. Mol Cell. 2020;77(2): 213-227 e215.

11. Blomme A, Ford CA, Mui E, Patel R, Ntala C, Jamieson LE, et al. 2,4-dienoyl-CoA reductase regulates lipid homeostasis in treatment-resistant prostate cancer. Nat Commun. 2020;11(1): 2508.

12. Loftfield E, Rothwell JA, Sinha R, Keski-Rahkonen P, Robinot N, Albanes D, et al. Prospective Investigation of Serum Metabolites, Coffee Drinking, Liver Cancer Incidence, and Liver Disease Mortality. J Natl 
Cancer Inst. 2020;112(3): 286-294.

13. Turkoglu O, Zeb A, Graham S, Szyperski T, Szender JB, Odunsi K, et al. Metabolomics of biomarker discovery in ovarian cancer: a systematic review of the current literature. Metabolomics. 2016;12(4).

14. Kozar N, Kruusmaa K, Bitenc M, Argamasilla R, Adsuar A, Goswami N, et al. Metabolomic profiling suggests long chain ceramides and sphingomyelins as a possible diagnostic biomarker of epithelial ovarian cancer. Clin Chim Acta. 2018;481: 108-114.

15. Yang W, Mu T, Jiang J, Sun Q, Hou X, Sun Y, et al. Identification of Potential Biomarkers and Metabolic Profiling of Serum in Ovarian Cancer Patients Using UPLC/Q-TOF MS. Cell Physiol Biochem. 2018;51(3): 1134-1148.

16. Plewa S, Horala A, Derezinski P, Nowak-Markwitz E, Matysiak J, Kokot ZJ. Wide spectrum targeted metabolomics identifies potential ovarian cancer biomarkers. Life Sci. 2019;222: 235-244.

17. Govorov I, Sitkin S, Pervunina T, Moskvin A, Baranenko D, Komlichenko E. Metabolomic Biomarkers in Gynecology: A Treasure Path or a False Path? Curr Med Chem. 2020;27(22): 3611-3622.

18. Bachmayr-Heyda A, Aust S, Auer K, Meier SM, Schmetterer KG, Dekan S, et al. Integrative Systemic and Local Metabolomics with Impact on Survival in High-Grade Serous Ovarian Cancer. Clin Cancer Res. 2017;23(8): 2081-2092.

19. Zennaro L, Nicole L, Vanzani P, Cappello F, Fassina A. (1)H-NMR spectroscopy metabonomics of reactive, ovarian carcinoma and hepatocellular carcinoma ascites. Pleura Peritoneum. 2020;5(2): 20200113.

20. Siegel RL, Miller KD, Jemal A. Cancer statistics, 2019. CA Cancer J Clin. 2019;69(1): 7-34.

21. Chen RR, Yung MMH, Xuan Y, Zhan S, Leung LL, Liang RR, et al. Targeting of lipid metabolism with a metabolic inhibitor cocktail eradicates peritoneal metastases in ovarian cancer cells. Commun Biol. 2019;2: 281.

22. Butterfield JH. Survey of Mast Cell Mediator Levels from Patients Presenting with Symptoms of Mast Cell Activation. Int Arch Allergy Immunol. 2020;181(1): 43-50.

23. Alves MR, Do Amaral NS, Marchi FA, Silva FIB, Da Costa A, Carvalho KC, et al. Prostaglandin D2 expression is prognostic in highgrade serous ovarian cancer. Oncol Rep. 2019;41(4): 2254-2264.

24. Li Y, Kasim V, Yan X, Li L, Meliala ITS, Huang C, et al. Yin Yang 1 facilitates hepatocellular carcinoma cell lipid metabolism and tumor progression by inhibiting PGC-1beta-induced fatty acid oxidation. Theranostics. 2019;9(25): 7599-7615.

25. Mika A, Kobiela J, Czumaj A, Chmielewski M, Stepnowski P, Sledzinski T. Hyper-Elongation in Colorectal Cancer Tissue - Cerotic Acid is a Potential Novel Serum Metabolic Marker of Colorectal Malignancies. Cell Physiol Biochem. 2017;41(2): 722-730.

26. Prusinkiewicz MA, Gameiro SF, Ghasemi F, Dodge MJ, Zeng PYF, Maekebay H, et al. Survival-Associated Metabolic Genes in Human Papillomavirus-Positive Head and Neck Cancers. Cancers (Basel). 2020;12(1).

27. Ke C, Li A, Hou Y, Sun M, Yang K, Cheng J, et al. Metabolic phenotyping for monitoring ovarian cancer patients. Sci Rep. 2016;6: 23334. 
28. Fu T, Coulter S, Yoshihara E, Oh TG, Fang S, Cayabyab F, et al. FXR Regulates Intestinal Cancer Stem Cell Proliferation. Cell. 2019;176(5): 1098-1112 e1018.

29. Jeon SM, Shin EA. Exploring vitamin D metabolism and function in cancer. Exp Mol Med. 2018;50(4): 20.

30. Nazih H, Bard JM. Cholesterol, Oxysterols and LXRs in Breast Cancer Pathophysiology. Int J Mol Sci. 2020;21(4).

31. Suh S, Kim KW. Diabetes and Cancer: Cancer Should Be Screened in Routine Diabetes Assessment. Diabetes Metab J. 2019;43(6): 733-743.

32. Archid R, Solass W, Tempfer C, Konigsrainer A, Adolph M, Reymond MA, et al. Cachexia Anorexia Syndrome and Associated Metabolic Dysfunction in Peritoneal Metastasis. Int J Mol Sci. 2019;20(21).

33. Lyu J, Yang EJ, Head SA, Ai N, Zhang B, Wu C, et al. Astemizole Inhibits mTOR Signaling and Angiogenesis by Blocking Cholesterol Trafficking. Int J Biol Sci. 2018;14(10): 1175-1185.

\section{Figures}

A

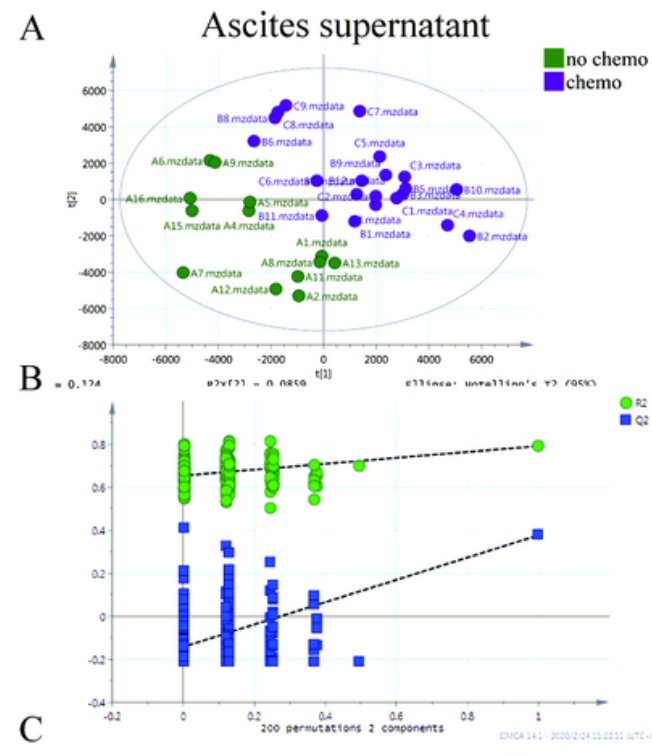

$\mathrm{C}$

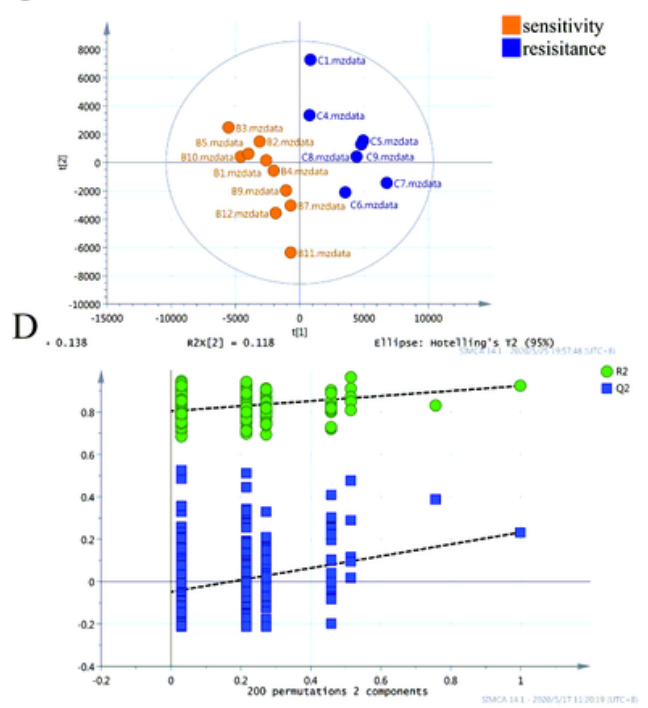

Precipitation cell
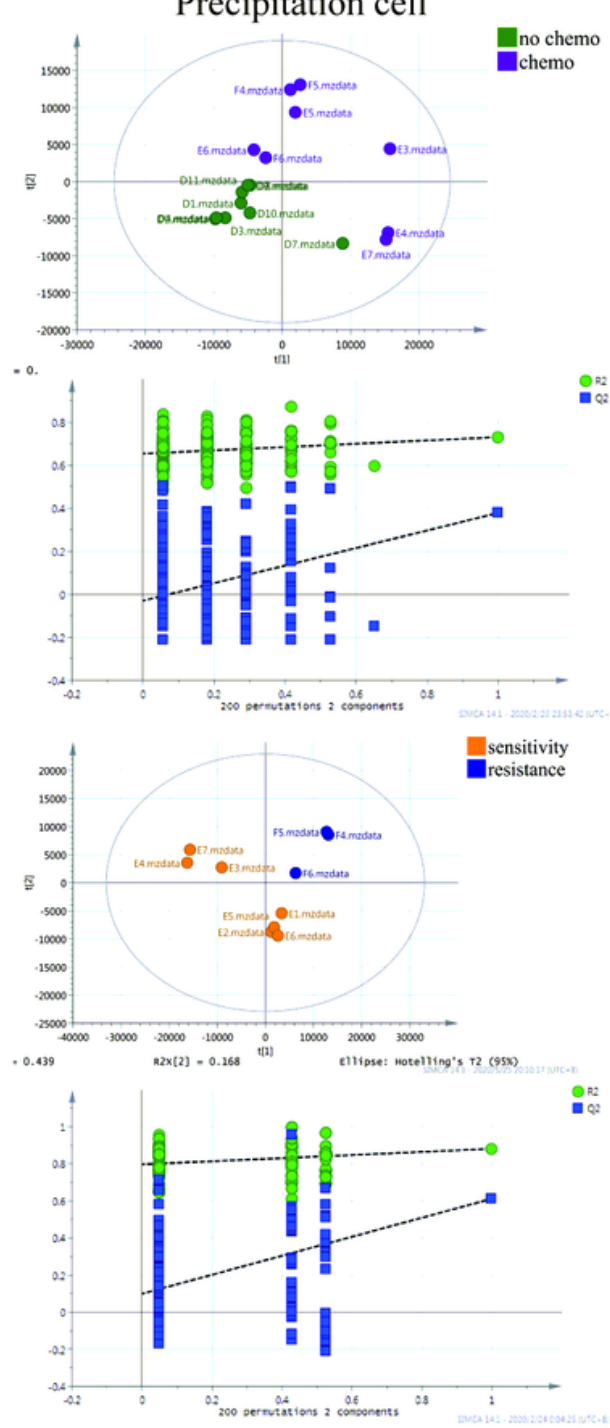

no chemo
chemo
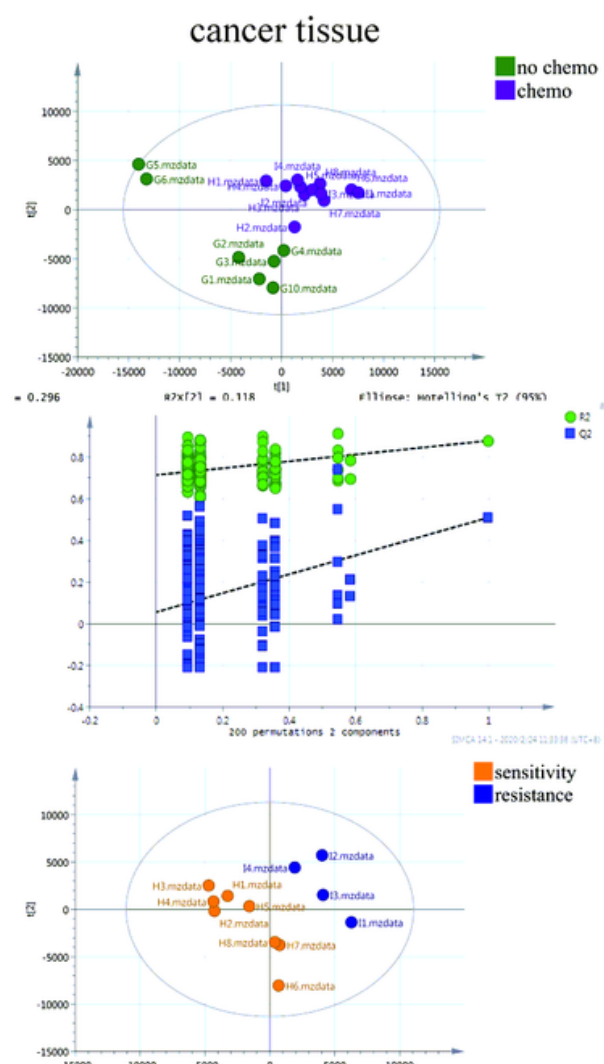

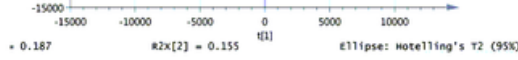

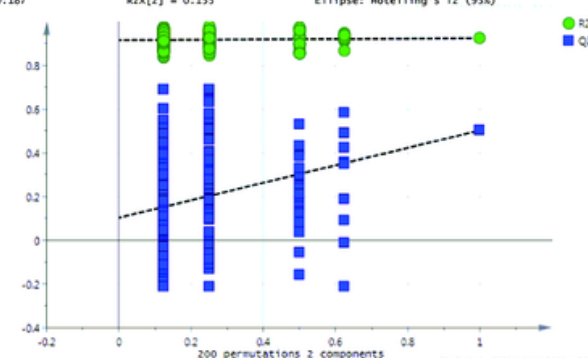


Results of supervised partial least squares discriminant analysis (PLS-DA) and permutation test. a-b. No chemotherapy and chemotherapy groups. c-d. chemosensitivity and chemotherapy resistance groups.

Ovarian cancer ascites supernatant, precipitated cells and tissue samples can be separated. The permutation test indicates that the PLS-DA model of tissue data set is not over-fitting.
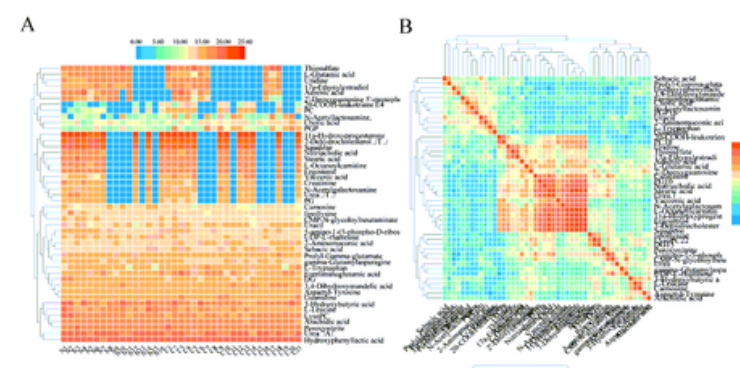

C

D
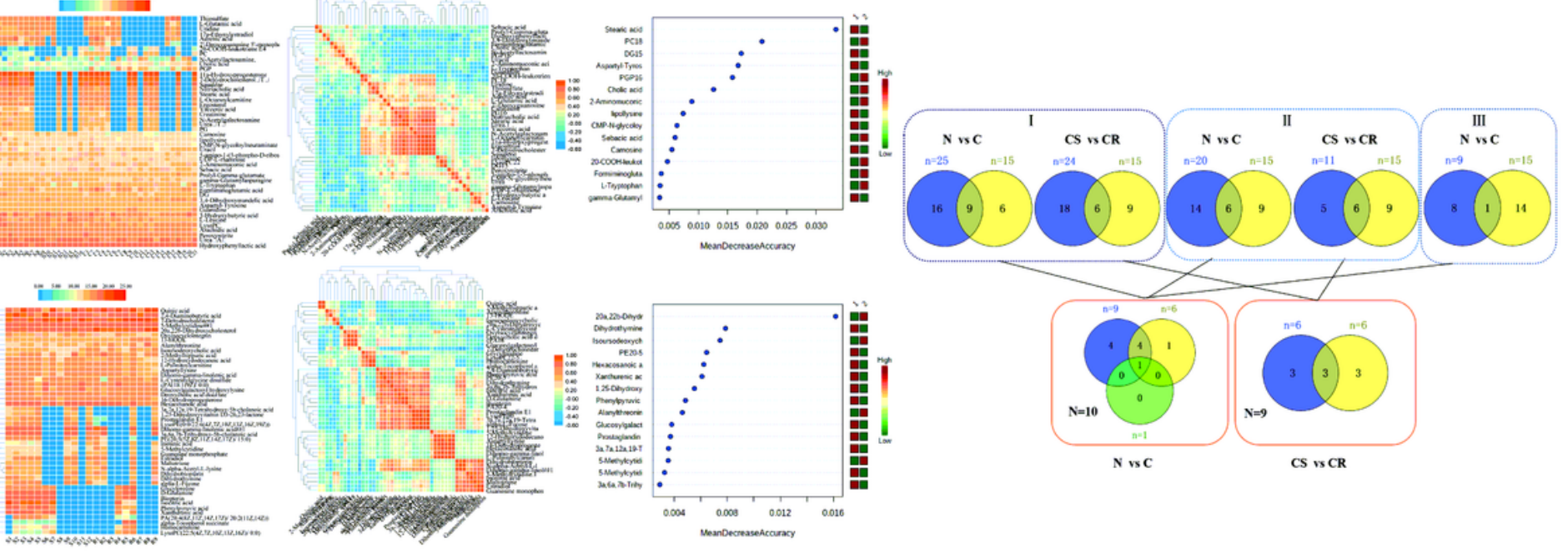

\section{Figure 2}

Metabolites Characteristics of ascites supernatant, precipitated cells and tissues in ovarian cancer. a. Results of hierarchical cluster analysis of metabolites of ascites supernatant (nasty 37), precipitated cells (nasty 23) and cancer tissue (naphtha 22) in ovarian cancer. Each row represents a metabolite, and each column represents a different group ( $\mathrm{N}$ vs $\mathrm{C}$ and $\mathrm{CS}$ vs $\mathrm{CR}$ ). b. Correlation heatmap of differential metabolites. $\mathrm{C}$. Random forests show important differential metabolites in N vs C (up) and CS vs CR (down). d. The analysis flow of screening differential metabolites. 
A
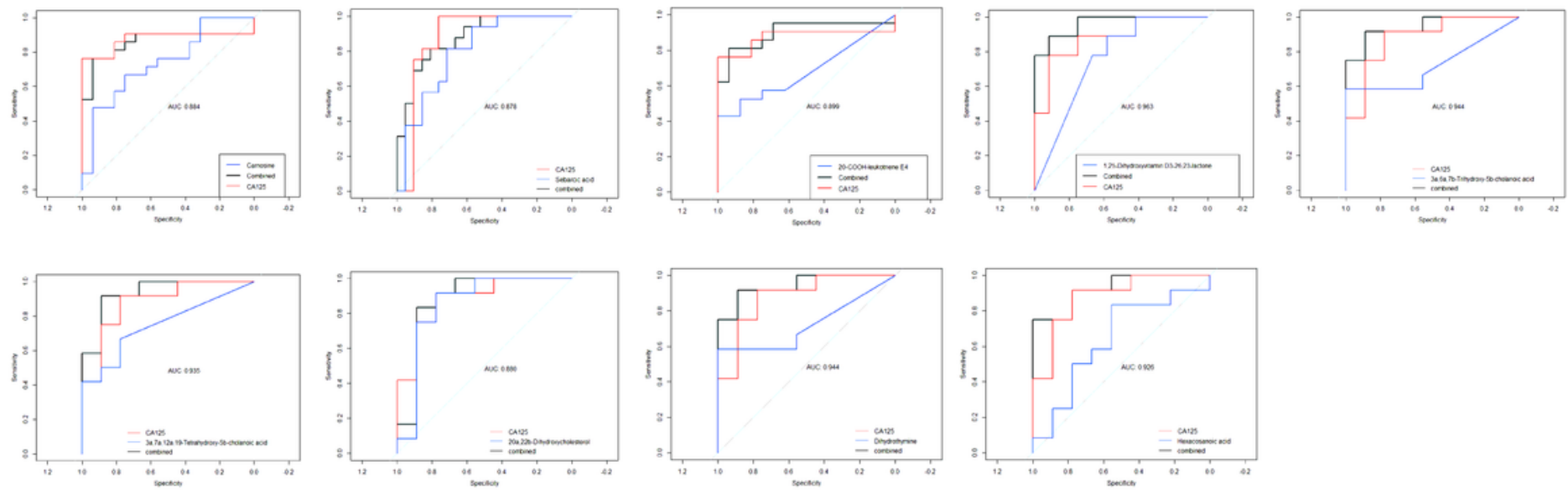

B
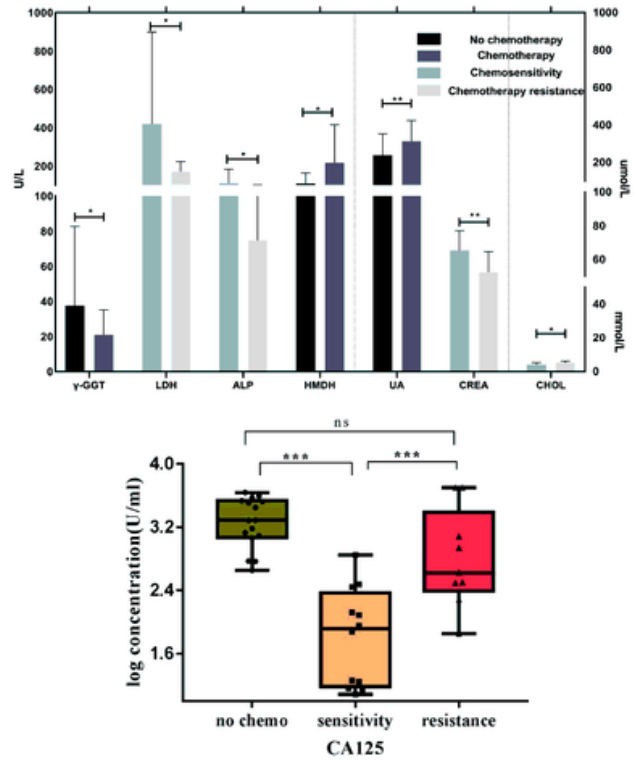

$\mathrm{C}$
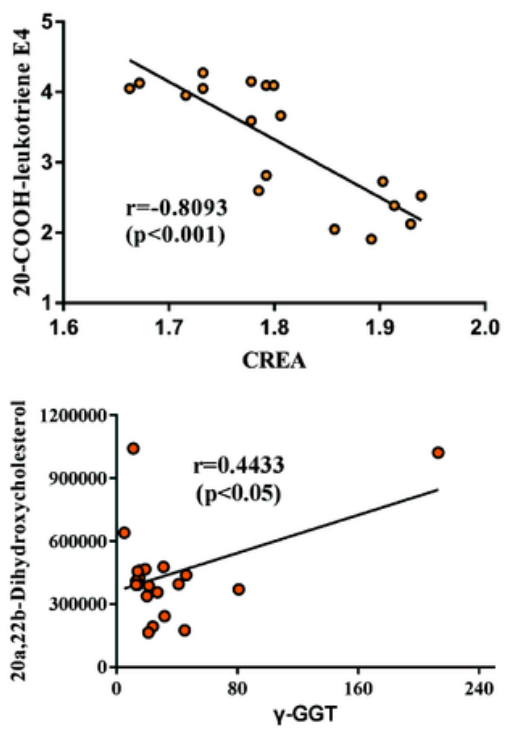

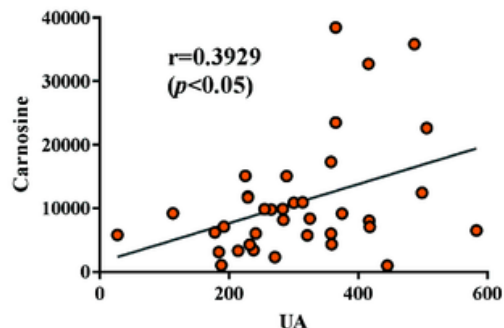

UA

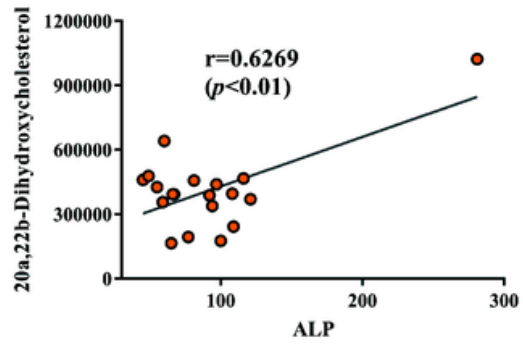

Figure 3

Analysis of differential metabolites and clinical data of patients. a. ROC curves of 9 metabolites combined with CA125 respectively. b. The expression of serological markers in $N$ vs $C$ and CS vs $C R(p<0.05)$. The ordinate is the concentration (log). c. Correlation analysis scatter plot for 20-COOH-LTE4 and creatinine, carnosine and uric acid, 20a,22 b-dihydroxycholesterol and $y-G G T, A L P(p<0.05)$. 
A
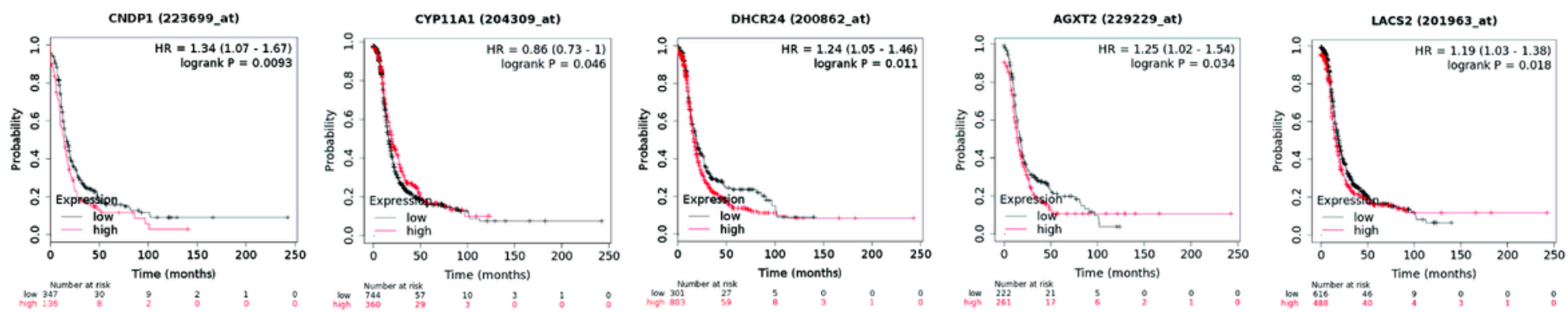

$\mathrm{B}$

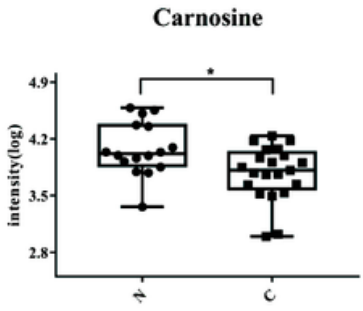

20a,22b-Dihydroxycholesterol

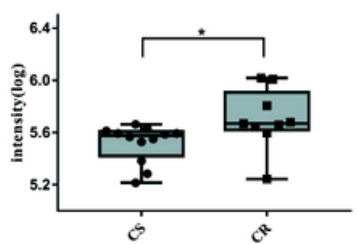

1,25-Dihydroxyvitamin D326,23-lactone

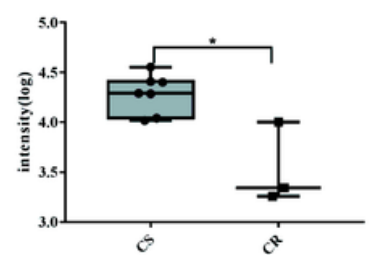

Dihydrothymine

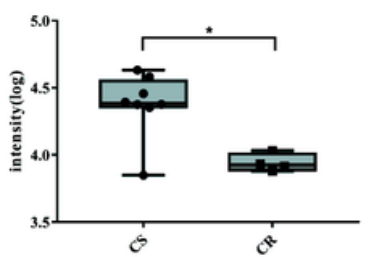

Hexacosanoic acid

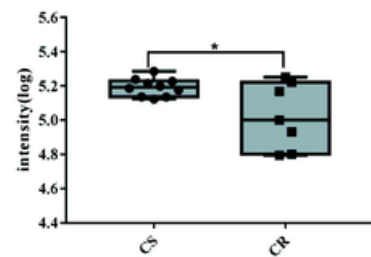

Figure 4

Expression of differential metabolites and correlation between related enzyme genes and progression-free survival of patients. a. Progression-free survival curve of differential metabolites $(p<0.05)$. b. Box plots of corresponding metabolite biomarkers in in N vs C (blank) and CS vs CR (gray) samples $(p<0.05)$.

A

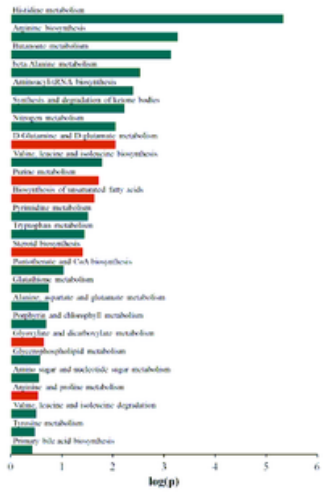

B

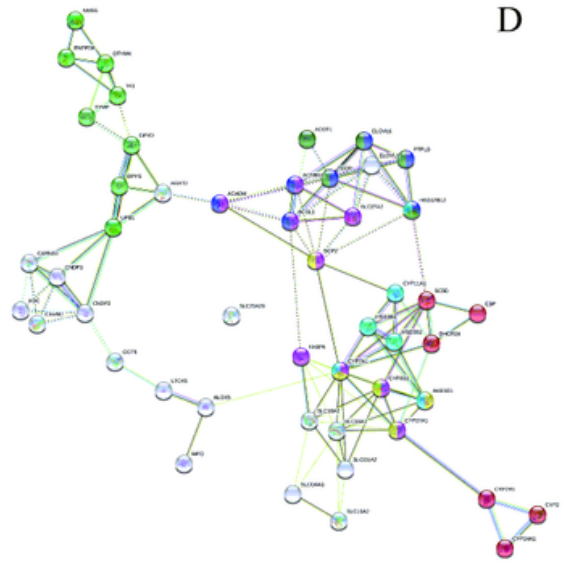

C
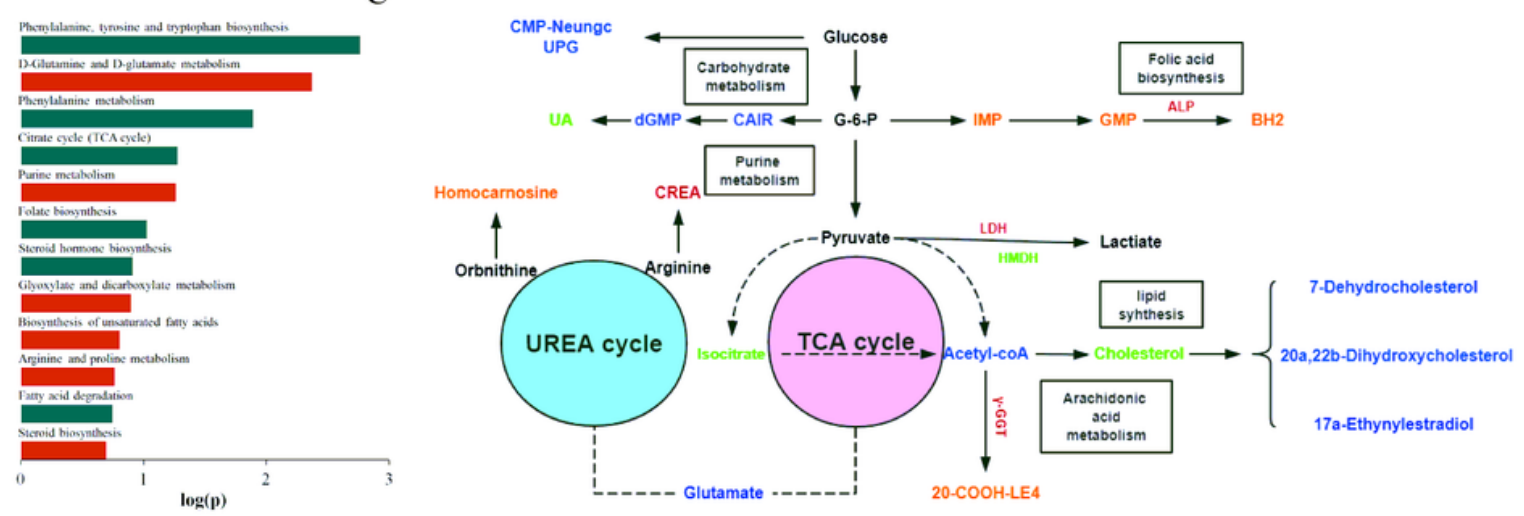

Figure 5 
Pathway analysis of differential metabolites between ovarian cancer tissue and ascites. a. Enrichment pathway bar chart of differential metabolites (orange is shared pathway) in N vs C (left), CS vs CR (right) groups. b. The shared pathways involved in differential metabolites and serological indicators. c. Network view of STRING analysis of genes related to differential metabolites $(p<0.001)$. $d$. Proposed metabolic mechanisms associated with ovarian cancer. Red font represents the $\mathrm{N}$ vs $\mathrm{C}$ group and blue font represents the CS vs CR group. and denote down-regulation/up-regulation.

\section{Supplementary Files}

This is a list of supplementary files associated with this preprint. Click to download.

- Supplementaryfiture2.tif

- Supplementaryfigure1.tif

- SupplementarytableS5.xIsx

- SupplementarytableS4.xlsx

- SupplementarytableS3.xlsx

- SupplementarytableS2.xIsx

- SupplementarytableS1.xlsx

- Supplementalinformation.docx 